\title{
The Ether Theory Unifying the Relativistic Gravito-Electromagnetism Including also the Gravitons and the Gravitational Waves
}

\author{
David Zareski ${ }^{1}$ \\ ${ }^{1}$ I.A.I, Israel Aerospace Industries, Yehud, Israel \\ Correspondence: David Zareski, I.A.I, Israel Aerospace Industries, Yehud, Israel. E-mail: zareski@inter.net.il
}

Received: April 18, 2017

Accepted: April 28, 2017

Online Published: May 12, 2017

doi:10.5539/apr.v9n3p21

URL: https://doi.org/10.5539/apr.v9n3p21

\begin{abstract}
In previous publications, we showed that Maxwell's equations are an approximation to those of General Relativity when $V \ll c$, where $\boldsymbol{V}$ is the velocity of the particle submitted to the electromagnetic field. This was demonstrated by showing that the Lienard-Wiechert potential four-vector $A_{\mu}$ created by an electric charge is the equivalent of the gravitational four-vector $G_{\mu}$ created by a massive neutral point when $V \ll c$.

In the present paper, we generalize these results for $\boldsymbol{V}$ non-restricted to be small. To this purpose, we show first that the exact Lagrange-Einstein function of an electric charge $q$ submitted to the field due an immobile charge $q_{0}$ is of the same form as that of a particle of mass $m$ submitted to the field created by an immobile particle of mass $m_{0}$. Maxwell's electrostatics is then generalized as a case of the Einstein's general relativity. In particular, it appears that an immobile $q_{0}$ creates also an electromagnetic horizon that behaves like a Schwarzschild horizon. Then, there exist ether gravitational waves constituted by gravitons in the same way as the electromagnetic waves are constituted by photons.

Now, since $A_{\mu}$ and $G_{\mu}$, are equivalent, and as we show, $G_{\mu}$ produces the approximation, for $V \ll c$, of $g_{\mu 4}$ created by $m_{0}$ mobile, where the $g_{\mu v}$ are the components of Einstein's fundamental tensor, it follows that $A_{\mu}+$ $G_{\mu}$ produces the approximation, for $V \ll c$, of $\beth_{\mu 4}$, where the $\beth_{\mu \nu}$ created by $m_{0}$ and by $q_{0}$, generalize the $g_{\mu \nu}$.

Résumé. Dans des publications antérieures nous montrâmes que l'électromagnétisme de Maxwell est une approximation de la Relativité Générale quand $V \ll c$, ou $\boldsymbol{V}$ est la vitesse de la particule soumise au champ électromagnétique. Ceci a été prouvé en montrant que le quatre-vecteur potentiel de Lienard-Wiechert $A_{\mu}$ créé par une charge électrique est l'équivalent du quatre-vecteur gravitationnel $G_{\mu}$ créé par une masse ponctuelle neutre quand $V \ll c$.

Dans le présent article, nous généralisons ces résultats pour $\boldsymbol{V}$ non-restreinte à être petite. A cette fin, nous montrons d'abord que la fonction exacte de Lagrange-Einstein créé par une charge électrique $q$ soumise au champ créé par une charge électrique $q_{0}$ immobile est de la même forme que celle d'une particule de masse $m$ soumise au champ créé par une particule immobile de masse $m_{0}$. L'électrostatique de Maxwell est donc généralisée comme étant un cas de la relativité générale d'Einstein. En particulier, il apparait qu'une $q_{0}$ immobile crée aussi un horizon électromagnétique qui se conduit comme un horizon de Schwarzschild. Puis qu'il existe des ondes gravitationnelles constituées de gravitons de la même façon que les ondes électromagnétiques sont constituées de photons.

Or, puisque $A_{\mu}$ and $G_{\mu}$ sont équivalents, et que, comme nous le montrons, $G_{\mu}$ produit l'approximation de $g_{\mu 4}$ pour $V \ll c$, due a $m_{0}$ mobile, ou les $g_{\mu \nu}$ sont les composants du tenseur fondamental d'Einstein, il s'en suit que $A_{\mu}+G_{\mu}$ produit l'approximation de $\beth_{\mu 4}$, pour $V \ll c$, ou les $\beth_{\mu \nu}$ créés par $m_{0}$ et par $q_{0}$ généralisent les $g_{\mu \nu}$.
\end{abstract}

Keywords: Relativistic electromagnetism, completion of Einstein's relativity theory, gravitons and photons

\section{Introduction}

In Zareski (2014) and in Sec. IX of Zareski (2015), we showed, in particular, that from the elastic ether theory it appears that the form of Maxwell's Electromagnetism emerges as an approximation of General Relativity. The main lines of this demonstration were the following. Einstein's fundamental tensor of components $g_{\mu \nu}$, is the solution of the system of equations (44) of Einstein (1916) obtained from pure mathematical reasoning not related 
a priori only to gravitation. This is the reason why, in my opinion, the title of his famous paper: "The foundation of the general theory of relativity", do not comport the word 'gravitation'. Therefore, one can suppose that these equations are more general than defining only the gravitational field, and could define also other fields as the electromagnetic field.

Indeed, in Zareski (2014) and in Sec. IX of Zareski (2015), we have shown this fact in the case where the velocity $\boldsymbol{V}$ of the particle is such that $V \ll c$, that is to say that we have shown that Maxwell's electromagnetism is of the same form as Newton's gravitation. This was shown as following.

a) First, we showed that Coulomb's electrostatic potential $A_{4, S}$, (s for static), created by an immobile electric charge, is of the same form as the Newton gravitostatic potential $G_{4, S}$ created by an immobile neutral massive particle.

b)Then, we generalized the result a) for $V \ll c$, by showing that the Lienard-Wiechert potential four-vector $A_{\mu}$ created by a moving electric charge, differs from the gravitational four-vector $G_{\mu}$ created by a moving massive point by only a constant multiplicative coefficient.

From a) and b), it appears that for non-small $V$, Maxwell's electromagnetism could be generalized as a case of Einstein's general relativity. In the present paper, the result a) is indeed generalized for $V$ non-restricted to be small. That is to say that we show that the exact Lagrange-Einstein function of an electric charge $q$ submitted to the field due an immobile charge $q_{0}$ and its motion equation are the same as those of a particle of mass $m$ submitted to only the field created by an immobile particle of mass $m_{0}$. That is, we generalized Maxwell's electrostatic to a case of Einstein's general relativity. In particular, it appears that an immobile $q_{0}$ creates also an electromagnetic horizon that behaves like a Schwarzschild horizon, that is, when another electric charge $q$ is attracted by $q_{0}$ and reaches this electromagnetic horizon, then its velocity is there null. Then that there exist ether gravitational waves constituted of gravitons in the same way as the electromagnetic waves are constituted by photons.

Then we arrive to the conclusion that, since in the approximation $V \ll c, A_{\mu}$ and $G_{\mu}$ differs by only a constant multiplicative coefficient and since as we show, $G_{\mu}$ produces then the approximation of $g_{\mu 4}$, where one recalls that the $g_{\mu \nu}$ are the components of Einstein's fundamental tensor created by $m_{0}$ whether it moves or not, it follows that $A_{\mu}+G_{\mu}$ produces the approximation for $V \ll c$ of $\beth_{\mu 4}$, where the $\beth_{\mu \nu}$ are the components of a tensor that generalizes Einstein's fundamental tensor $g_{\mu \nu}$ by taking into account the contribution of the electrical charge $q_{0}$.

\section{Notations and Recalls}

\subsection{Some Generalities}

The Greek indexes take the values 1,2,3,4 and the Latin the values 1,2,3 the index 4 corresponding to ct. The low indexes are covariant while the high are contravariant. We use the Einstein summation, the time derivative $d y / d t$ of $\mathrm{y}$ is denoted simply $\dot{y}$. A particle of mass $\mathrm{m}$ and electric charge $\mathrm{q}$ will be denoted $P(m, q)$. $g_{\mu \nu}$ denotes Einstein's tensor which is a solution of the system of equations (44) of Einstein (1916). In the present paper, we do not take into account dark matter since we consider only individual particles. The velocity of the free light is denoted $c$, and the constant of gravitation is denoted $\mathrm{k}$. The coordinates origin is denoted $\mathbf{O}$.

The Lagrange-Einstein function $L_{G}$ of a $P(m, 0)$ submitted to a field $g_{\mu \nu}$ is

$$
L_{G}=-m c \dot{S}
$$

where

$$
\dot{s}=\sqrt{g_{\mu \nu} \dot{x}^{\mu} \dot{x}^{v}}
$$

and the motion equation of this particle Cf. e.g., Zareski (2012), or Eq. (3.3) of Zareski (2015), is

$$
\frac{d}{d t}\left(\frac{\partial}{\partial x^{\mu}} L_{G}\right)-\partial_{\mu} L_{G}=0
$$

The field created by an immobile $P\left(m_{0}, 0\right)$ located at $\mathbf{O}$, is of Schwarzschild, and $L_{G}$ denoted then $L_{G S G}$ the indexes $S G$ referring to static-gravitation, is then

$$
L_{G S G}=-m c \sqrt{c^{2} \gamma^{2}-V^{2} \gamma_{\vartheta}^{2}}
$$

where, Cf., e.g., Zareski (2012), or Eq. (3.62) and (3.63) of Zareski (2015),

$$
\gamma^{2} \equiv 1-\alpha / r
$$




$$
\gamma_{\vartheta}^{2} \equiv 1+\alpha\left(\cos ^{2} \vartheta\right) /\left(r \gamma^{2}\right)
$$

where $\alpha$ is the constant defined by

$$
\alpha=2 m_{0} k / c^{2},
$$

and $\vartheta$ denotes the angle made by the velocity $V$ of this $P(m, 0)$ and the radius vector $r$ issued from $\mathrm{O}$. Inserting (6) into (4), one obtains

$$
L_{G S G}=-m c \sqrt{c^{2}(1-\alpha / r)-V^{2}\left(1+\alpha\left(\cos ^{2} \vartheta\right) /(r-\alpha)\right)}
$$

and, Cf. Eq. (3.68) of Zareski (2015), the expression for the absolute value of the velocity $V$ of this $P(m, 0)$ of total energy $E_{T}$ is

$$
V=c\left(\gamma / \gamma_{\vartheta}\right) \sqrt{1-\left(\gamma m c^{2} / E_{T}\right)^{2}}
$$

The motion equation is then given by Eq. (3) with $L_{G S G}$ instead of $L_{G}$. Furthermore, Eq. (9) shows that $V=0$ at $r=\alpha$, i.e., the $P(m, 0)$ stops on the Schwarzschild horizon (SH).

Now, if $V_{r}(r)$ denotes the expression for $V$ when the trajectory of this $P(m, 0)$ is a ray issued from $\mathbf{O}$, that is, when $\vartheta=0$, then (9) becomes

$$
V_{r}(r)=c(1-\alpha / r) \sqrt{1-(1-\alpha / r)\left(m c^{2} / E_{T}\right)^{2}}
$$

\subsection{Dirac's Considerations Regarding the $P(m, 0)$ in a Schwarzschild Field}

Expression (10) is of the same form as the absolute Dirac expression for the radial velocity of this $P(m, 0)$ as it appears in the fifth equation of page 33 of Dirac's book. Cf. Dirac (1975). Indeed, he wrote there that the expression $d r / d t$ for the radial velocity directed toward the $\mathrm{SH}$, of this $P(m, 0)$ is

$$
d r / d t=-c\left(1-\frac{2 M}{r}\right) \sqrt{1-\left(1-\frac{2 M}{r}\right) / k_{D}^{2}} .
$$

In this equation I use the notation $M$ and $k_{D}$ instead of $m$ and of $k$ used by Dirac, and add $c$ that missed in its original expression, the sign $(-)$ is due to the fact that Dirac considered that the motion is directed toward the origin. We see that, if one writes

$$
2 M \equiv \alpha, \quad M \equiv m_{0} k / c^{2}, \quad m c^{2} / E_{T} \equiv 1 / k_{D},
$$

the expression for $|d r / d t|$ is identical to that for $V_{r}(r)$. Furthermore, using Dirac's reasoning, let us demonstrate that the time taken by the $P(m, 0)$ to reach the $\mathrm{SH}$ is infinite, i.e., near the $\mathrm{SH}$, the velocity of the particle is very small, and null on it.

Indeed, near the $\mathbf{S H}$, the term $(1-\alpha / r)\left(m c^{2} / E_{T}\right)^{2}$ is negligible in front of 1 since $(1-\alpha / r)$ tends then toward 0 , that is to say that in this case, Eq. (11) yelds

which yields, after integration:

$$
d t \cong-\alpha d r /[c(r-\alpha)],
$$

$$
t \cong(\alpha / c) \log [1 /(r-\alpha)]+\text { const }
$$

Equation (12) shows that the $P(m, 0)$ reaches the SH after an infinitely long time measured by an immobile clock. It appears that our unifying ether theory englobes the Dirac theory regarding the behavior of the particle in a Schwarzschild field for $r \geq \alpha$. In the frame of this unification we do not treat the case where particles could be located into the black hole.

\subsection{The Newton Motion Equation as Approximation of the Real Motion Equation}

The exact motion equation of the $P(m, 0)$ in the Schwarzschild field is given by Eq. (3) in which $L_{G}$ is replaced by $L_{G S G}$ defined in Eq. (8). That is, this exact motion equation is

$$
-m c\left\{\frac{d}{d t}\left[\frac{\partial}{\partial x^{\mu}} \sqrt{c^{2} \gamma^{2}-V^{2} \gamma_{\vartheta}^{2}}\right]-\partial_{\mu} \sqrt{c^{2} \gamma^{2}-V^{2} \gamma_{\vartheta}^{2}}\right\}=0
$$

where $\gamma^{2}$ and $\gamma_{\vartheta}{ }^{2}$ are given by (5)-(7). Now, with an evident notation, it appears from (8) that

$$
L_{G S G}(V \ll c \text { and } r \gg \alpha)=-m c \sqrt{c^{2}-V^{2}}+m c^{2} \alpha /(2 r),
$$

and when one inserts $L_{G S G}(V \ll c$ and $r \gg \alpha)$ in (3) instead of $L_{G}$ one obtains instead of (13), the following approximated motion equation

$$
m \ddot{\boldsymbol{r}} \cong-m c^{2} \alpha \mathbf{r} /\left(2 r^{3}\right) \equiv-m m_{0} k \mathbf{r} / r^{3}
$$


Equation (15) is the approximated motion equation of a $P(m, 0)$ submitted to the gravitational field due to an immobile $P\left(m_{0}, 0\right)$.

\subsection{Some Recalls on the Elastic Interpretation of Maxwell's Equations}

Let us first recall Maxwell's opinion on the elastic interpretation of electromagnetism, he wrote in Art. 866 of Maxwell (1954)

"Hence all these theories lead to the conception of a medium in which the propagation takes place, and if we

admit this medium as an hypothesis, I think it ought to occupy a prominent place in our investigations.". And Einstein wrote Cf. Einstein (1920):

"According to the general theory of relativity space without ether is unthinkable......"

We recall now some results regarding the elastic interpretation of Maxwell's equations, presented in Zareski (2001), (rewritten in Zareski, 2015). There we considered a particular elastic medium, the 'ether', governed by the continuity equation (2) of Zareski (2001) and by the following equation of elasticity

$$
\operatorname{curl}\left(\boldsymbol{C} / 2-\eta_{0} \operatorname{curl} \xi\right)=\rho_{0} \partial_{t t} \xi
$$

that defines the elastic changes $\xi$ in the ether due to the density of couples of forces $\boldsymbol{C}$ applied to it, and from which one deduces the Maxwell equations of electromagnetism as recalled he below. In Eq. (16), $\eta_{0}$ denotes the inverse of the free induction coefficient, which, in the elastic interpretation, is the free elastic restoring rotation coefficient, and $\eta_{0}$ is related to $c$ by the relation

$$
\eta_{0}=\rho_{0} c^{2}
$$

where $\rho_{0}$ denotes the ether density. We recall in particular that by introducing the new variables $, \boldsymbol{H}, \boldsymbol{B}$, $J_{e}$, and $\rho_{e}$, defined by

$$
\begin{gathered}
\boldsymbol{E}=\eta_{0} \operatorname{curl} \boldsymbol{\xi}-\boldsymbol{C} /(2) \\
\boldsymbol{H}=\partial_{t} \xi, \quad \boldsymbol{B}=\rho_{0} \partial_{t t} \xi \\
\boldsymbol{J}_{\boldsymbol{e}}=\boldsymbol{\partial}_{\boldsymbol{t}} \boldsymbol{C} /\left(2 \eta_{0}\right), \quad \rho_{\boldsymbol{e}}=-\operatorname{div} \boldsymbol{C} /\left(2 \eta_{0}\right),
\end{gathered}
$$

and by eliminating $\xi$ and $\boldsymbol{C}$ between these equations, one obtains the Maxwell equations.

For example, from Eqs. (16), (18) and (20), one obtains

$$
\operatorname{curl} \boldsymbol{E}+\partial_{t} \boldsymbol{B}=\mathbf{0}
$$

and by differentiating Eq. (18) with respect of the time and taking into account Eqs. (19) and (21), one obtains

$$
\operatorname{curl} \boldsymbol{H}-\partial_{t} \boldsymbol{E} / \eta_{0}=\boldsymbol{J}_{\boldsymbol{e}} .
$$

Equations (23) and (24) are Maxwell's equations, the others being obtained in the same way.

Remark. Since an electrically charged particle musts possess a mass, it follows that the results of this Sec. 2.4, i.e., Maxwell's electromagnetism is only an approximation. In the exact case one has to take into account the mass of this electrically charged particle that is to say one has to take into account the General Relativity as done here below.

\section{Relativistic Electromagnetism and Gravitation Equivalence for a $P(m, q)$ in the Field Due to an} Immobile $P\left(m_{0}, q_{0}\right)$

\subsection{Generalities}

One recalls that since Einstein's Eqs. (44) of Einstein (1916) were obtained by pure mathematical reasoning, one can suppose that these equations are more general than defining only the gravitational field. Indeed, as we show now, they define also the exact electromagnetic equations that complete the Maxwell equations shown to be an approximation, by taking into account the general relativity.

We generalize now first Maxwell's electrostatic as a case of the Einstein's static general relativity and show that, in particular, an immobile $q_{0}$ creates also an 'electromagnetic horizon' that behaves like a SH, that is to say that, when another electric charge $q$ is attracted by $q_{0}$ and reaches this electromagnetic horizon, then its velocity is there null. In that context, we generalize first the value of the constant $\alpha$.

Remark. From here on, we use the notation $\alpha_{G}, \gamma_{G}, \gamma_{G \vartheta}$ instead of $\alpha, \gamma, \gamma_{\vartheta}$, the index $G$ referring to gravitation. 


\subsection{Generalization of the Constant $\alpha_{G}$ for the Static Gravito-Electromagnetic Case}

When a $P(m, q)$ is submitted to an electrostatic field created by a $q_{0}$, it is also submitted to a gravitational field due to the mass $m_{0}$ of the charge $q_{0}$, since an electric particle possess also a mass. It follows that, when a $P(m, q)$ is submitted to the total field due to the immobile $P\left(m_{0}, q_{0}\right)$, that is, to the sum of the gravitational field due to $m_{0}$ and of the electrostatic field due to $q_{0}$, then, the approximation $L_{G S T}\left(V \ll c\right.$ and $\left.r \gg \alpha_{T}\right)$ of the Lagrange-Einstein $L_{G S T}$, the index $S T$ referring to static total fields, is then

$$
L_{G S T}\left(V \ll c \text { and } r \gg \alpha_{T}\right)=-m c \sqrt{c^{2}-V^{2}}+\left(-\frac{q q_{0}}{4 \pi \varepsilon_{0}}+m m_{0} k\right) \frac{1}{r} .
$$

Now, let the constant $\alpha_{E}$ be defined by

$$
\alpha_{E}=-\frac{2}{c^{2}(m / q)} \frac{q_{0}}{4 \pi \varepsilon_{0}}
$$

the constant $\alpha$ be denoted now $\alpha_{G}$ that is Cf. Eq. (7),

and let the constant $\alpha_{T}$ be defined by

$$
\alpha_{G}=2 m_{0} k / c^{2}
$$

$$
\alpha_{T}=\alpha_{G}+\alpha_{E}
$$

With these notations, (25) and the non-relativistic motion equation can be written

$$
\begin{gathered}
L_{G S T}\left(V \ll c \text { and } r \gg \alpha_{T}\right)=-m c \sqrt{c^{2}-V^{2}}+\frac{m c^{2}}{2} \alpha_{T} \frac{1}{r}, \\
m \ddot{\boldsymbol{r}} \cong-\frac{m c^{2}}{2} \alpha_{T} \frac{1}{r^{3}} \boldsymbol{r} .
\end{gathered}
$$

Note: For elementary electrically charged particles, then in general, as shown, e.g., in Zareski (2015), $m m_{0} k \ll$ $\left|q q_{0}\right| / 4 \pi \varepsilon_{0}$, therefore, in this non-relativistic electrostatic case, (30) can be written. $m \ddot{\mathbf{r}} \cong \frac{q q_{0}}{4 \pi \varepsilon_{0}} \frac{1}{r^{3}} \boldsymbol{r} \equiv q \boldsymbol{E}$, where $\boldsymbol{E}=\frac{q_{0}}{4 \pi \varepsilon_{0}} \frac{1}{r^{3}} \boldsymbol{r}$ denotes the electrical field created by $q_{0}$.

3.3 Exact Motion Equation and Velocity of a $P(m, q)$ due to an Immobile $P\left(m_{0}, q_{0}\right)$

This exact relativistic motion equation of a $P(m, q)$ in the field of an immobile $P\left(m_{0}, q_{0}\right)$ is

$$
\left(\frac{d}{d t} \frac{\partial}{\partial x^{j}}-\frac{\partial}{\partial x^{j}}\right) L_{G S T}=0
$$

the Lagrange-Einstein function $L_{G S T}$ being defined by

where $\dot{s}_{S T}$ is defined by

$$
L_{G S T}=-m c \dot{S}_{S T},
$$

and, $\gamma_{T}$ and $\gamma_{\vartheta T}$ by

$$
\dot{S}_{S T}=\sqrt{c^{2}{\gamma_{T}}^{2}-V^{2} \gamma_{\vartheta T}{ }^{2}},
$$

$$
{\gamma_{T}}^{2} \equiv 1-\alpha_{T} / r, \quad \gamma_{\vartheta T}{ }^{2} \equiv 1+\alpha_{T}\left(\cos ^{2} \vartheta\right) /\left(r{\gamma_{T}}^{2}\right)
$$

In this generalized Schwarzschild case, the expression for the velocity $V$ given in Eq. (9) is generalized by $V_{T}$ defined by

$$
V_{T}=c\left(\gamma_{T} / \gamma_{\vartheta T}\right) \sqrt{1-\left(\gamma_{T} m c^{2} / E_{T}\right)^{2}} .
$$

From Eq. (35) it appears that $V_{T}=0$ at $r=\alpha_{T}$, that is, the $P(m, q)$ submitted to the static field due to $P\left(m_{0}, q_{0}\right)$ stops on the 'generalized Schwarzschild horizon', (GSH). When $m=0$, then, Cf. Eq. (3.12) and Eq. (3.14) of Zareski (2015) where $g_{44}$ is $\gamma_{T}{ }^{2}$,

$$
\lim _{m \rightarrow 0}\left(m / \dot{S}_{S T}\right)=h v /\left(c^{3} \gamma_{T}{ }^{2}\right) .
$$

and Eq. (35) becomes 


$$
\lim _{m \rightarrow 0}\left(V_{T}\right)=c \gamma_{T} / \gamma_{\vartheta T} .
$$

Equation (35) shows that even the photons stop on the GSH. In fact even $V_{T}$ defined in (35) does not depend upon $m$ near to the $G S H$ since there $\left(\gamma_{T} m c^{2} / E_{T}\right)^{2}$ is infinitly small.

3.4 Ether Changes, General Waves Due to an Immobile $P\left(m_{0}, q_{0}\right)$ and to Mobile $P(m, q) s$ that are Gravitons or Photons when $m=q=0$

Equation (16) refers to the particular case where we considered that the density of couples of forces $C$ is due to only electric charges that create the electromagnetic field to which is submitted another electric charge. But here we generalize this case to the one where $\boldsymbol{C}$ denoted now $\boldsymbol{C}_{\boldsymbol{T}}$ is due also to the mass of massive neutral or electrically charged particles, remembering that an electrically charged particle must have a mass. That is, in presence of $\boldsymbol{C}_{T}$, the ether is such that the phase velocity $V_{P}$ propagated in it, is different from the light velocity $c$. Therefore in presence $\boldsymbol{C}_{\boldsymbol{T}}$, Eq. (16) has to be completed into the following

$$
\operatorname{curl}\left(C_{T} / 2-\eta \operatorname{curl} \xi\right)=\rho_{0} \partial_{t t} \xi
$$

where now

$$
\eta=\rho_{0} V_{P}^{2} .
$$

$\eta$ being the non-free $\eta_{0}$ since the phase velocity $V_{P}$ is different from $c$. From (38) and (39), one can obtain the generalized Maxwell equations as in Sec. 2.4.

In that context, let us first consider the field created by an immobile $P\left(m_{0}, q_{0}\right)$. The expression for the velocity $\boldsymbol{V}_{\boldsymbol{T}}$ of a $P(m, q)$ submitted to this field is given in (35) and the expression for the phase velocity $V_{P}$ associated to it, is, Cf. Eq. (3.69) of Zareski (2015),

$$
V_{p}=c \gamma_{T} /\left(\gamma_{\vartheta T} B_{T}\right)
$$

where

$$
B_{T} \equiv \sqrt{1-\left(\gamma_{T} m c^{2} / E_{T}\right)^{2}} .
$$

In particular, for $m=0$, then $B=1$, and (35) and (40) are identical, that is, the velocity of the photon, i.e., of the group velocity is identical to the phase velocity, i.e.,

$$
V_{P}(m=0)=V_{T}(m=0)=c \gamma_{T} / \gamma_{\vartheta T} .
$$

Since here one considers that the $P\left(m_{0}, q_{0}\right)$ is immobile at $\mathbf{O}$, it follows that at any point not situated at $\mathbf{O}$, (38) becomes

$$
-\operatorname{curl}\left(V_{P}^{2} \operatorname{curl} \xi\right)=\partial_{t t} \xi
$$

where $V_{P}$ is defined in (40) when $m \neq 0$ or in (42) when $m=0$. In the monochromatic case of pulsation $\omega$, (43) becomes

$$
\operatorname{curl}\left[\left(c \gamma_{T}\right)^{2} /\left(\gamma_{\vartheta T} B_{T}\right)^{2} \operatorname{curl} \xi\right]=\omega^{2} \xi
$$

in which, for the graviton, $m=0$, and $\alpha_{E}=0$, and for the photon, $m=0$, and $\alpha_{G}=0$.

We consider now the solutions of the equations of the ether changes (44) due to an immobile $P\left(m_{0}, q_{0}\right)$ in the two following cases: to $P\left(m_{0}, q_{0}\right)$ is submitted a $P(m, q)$, or a $P(0,0)$. To this purpose, let us consider the vector $\xi$ for which the expression is

where, when $m \neq 0, \phi$ is defined by

$$
\xi=\xi_{0} e^{i \phi},
$$

$$
\phi=\left(E_{T} / \hbar\right)\left(-t+\int d \ell / V_{P}\right)
$$

or by

$$
\phi=\left(E_{T} / \hbar\right)\left[-t+\int d \ell / V(m=0)\right],
$$

when $m=0$, and where $\xi_{0}$ is a vector depending only upon the spatial coordinates, Cf. Zareski (2012).

In Zareski (2016), we have shown that $\xi$ is the solution of Eq. (44) when the $P(m, q)$ describes a circle around $P\left(m_{0}, q_{0}\right)$, or a rectilinear trajectory directed toward it.

In Zareski (2013), and in Zareski (2015), Sec. IV. 2, we proved that, for sufficiently large $E_{T}$, then $\xi$ is the solution of Eq. (44) for any trajectory of the particle in the field created by a $P\left(m_{0}, q_{0}\right)$. 
The complete demonstration being laborious, one may suppose that $\xi$ defined in (45) and (46) is very close to the exact solution of (44) and might be the exact solution.

Now it appears that $\xi$, defined by (44) where one imposes $\left(c \gamma_{G}\right)^{2} /\left(\gamma_{{ }_{G}} B\right)^{2}$ instead of $\left(c \gamma_{T}\right)^{2} /\left(\gamma_{\vartheta T} B_{T}\right)^{2}$, by (45), and by (47), is a gravitational wave defined by

$$
\xi=\xi_{0} \exp \left\{i \omega\left[-t+\int d \ell \gamma_{\vartheta G} B / c \gamma_{G}\right]\right\} .
$$

If in order to fix the idea, one considers that the trajectory is rectilinear and passes by the immobile $P\left(m_{0}, q_{0}\right)$, then (48) becomes for large $r$

$$
\xi=\xi_{0} \exp \left[i \omega\left(-t+\frac{1}{c} \int \frac{d r}{1-\frac{2 m_{0} k}{c^{2} r}}\right)\right]
$$

Now, for very large $r$, then

$$
\xi \cong \xi_{0} \exp \left[i \omega\left(-t+\frac{r}{c}\right)\right]
$$

Equation (49) is a gravitation wave that for very very large $r$ becomes Eq. (50) which is of the same form as a free electromagnetic ether wave. From these equations (49) and (50), one deduces the equivalent Maxwell's equations for gravitation as done in Zareski (2001).

\subsection{Ether Globule Associated to the Particle and Creation of the Schwarzschild Field}

\subsubsection{Some General Recalls on the Ether Globule Associated to the Particle}

To fix the idea, let us consider a $P(M, Q)$ of constant velocity $\boldsymbol{V}(M, Q)$ directed along the $\mathrm{x}$-axe, to which is associated the phase velocity $\boldsymbol{V}_{\boldsymbol{P}}(M, Q)$, Cf. Eq. (21) of Zareski (2013), or Eq. (3.43) of Zareski (2015). Considering our above development, to this $P(M, Q)$ is associated a "single particle wave" $\widehat{\xi}(\Delta \omega)$ for which the expression is, Cf. Eq. (17) of Zareski (2014),

$$
\widehat{\xi}(\Delta \omega)=\xi_{0} \exp \left[i \omega\left(-t+\frac{x}{V_{\boldsymbol{P}}(M, Q)}\right)\right] \operatorname{SINC}\left[\frac{\Delta \omega}{2}\left(-t+\frac{x}{V(M, Q)}\right)\right]
$$

where $\operatorname{SINC}(y) \equiv(\sin y) / y$, and $\xi_{0}$ denotes a vector depending of only upon the spatial coordinates. This equation is another form of the following wave packet Cf. Eq. (18) of Zareski (2014),

$$
\widehat{\xi}(\Delta \omega)=\int_{\omega-\Delta \omega / 2}^{\omega+\Delta \omega / 2} \xi_{0} \exp \left[i \varpi\left(-t+\frac{x}{V_{P}(M, Q)}\right)\right] d \varpi .
$$

Equation (51) shows that:

$\widehat{\xi}(\Delta \omega)$ is a packet of waves, i.e., is a globule that moves with the velocity $\boldsymbol{V}(M, Q)$. In this globule,

the ether vibrates at the frequency $v=\omega /(2 \pi)$, and a wave moves with the phase velocity $\boldsymbol{V}_{\boldsymbol{P}}(M, Q)$.

3.5.2 The Globule Associated to the Free Gravitons or Photons

The photon and the graviton are $P(0,0)$, when they are free, (51) becomes

$$
\widehat{\xi}(\Delta \omega)_{\text {grav or phot }}=\xi_{0} \exp \left[i \omega\left(-t+\frac{x}{c}\right)\right] \operatorname{SINC}\left[\frac{\Delta \omega}{2}\left(-t+\frac{x}{c}\right)\right]
$$

This shows that the free graviton and the free photon move with the same velocity $\mathrm{c}$ which is also the phase velocity of the waves associated to them.

\subsubsection{Behaviour of the Immobile $\boldsymbol{P}(M, Q)$}

Let us consider what happens when the $\boldsymbol{P}(M, Q)$ is immobile, that is when $\boldsymbol{V}(M, Q)=0$. In this case $\boldsymbol{V}_{\boldsymbol{P}}(M, Q)$ is infinite, $E_{T}=m_{0} c^{2}$, and Cf. Eq. (22) of Zareski (2014), and Eq. (3.43) of Zareski (2015), (51) becomes

$$
\lim _{\boldsymbol{V}(M, Q) \rightarrow 0}[\hat{\xi}(\Delta \omega)]=\xi_{0} \exp \left(-i \frac{2 \pi}{h} m c^{2} t\right) \operatorname{SINC}\left[\frac{\Delta \omega}{2}\left(-t+\frac{x}{0}\right)\right] .
$$

Now, since $\operatorname{SINC}(\infty)=0$ because $\operatorname{SINC}\left[\frac{\Delta \omega}{2}\left(-t+\frac{x}{0}\right)\right]=0$, it follows that

$$
\lim _{\boldsymbol{V}(M, Q) \rightarrow 0}[\widehat{\xi}(\Delta \omega)]=0,
$$

that is to say that when the globule $\hat{\xi}(\Delta \omega)$ becomes immobile, it loses its form and becomes a Schwarzschild field since an immobile particle creates such a field. 


\section{Relativistic Electromagnetism and Gravitation Equivalence for a $P(m, q)$ in the Field Due to a $P\left(m_{0}, q_{0}\right)$ of Given Motion \\ 4.1 Approximation of the Lagrange-Einstein function of the $P(m, q)$ Submitted to the Field Due to a $P\left(m_{0}, q_{0}\right)$ of Given Motion}

Let $\boldsymbol{V}_{\mathbf{0}}$ denote the given velocity of a $P\left(m_{0}, q_{0}\right)$ that creates the field to which is submitted a $P(m, q)$, and let $W_{\mu}$ denote the tensor defined by

$$
W_{\mu} \equiv V_{0, \mu} /\left(r c-\boldsymbol{r} \cdot \boldsymbol{V}_{\mathbf{0}}\right)
$$

where the $V_{0, j}$ are the covariant components of $V_{0}$ and $V_{0,4}=c$. Let $L_{G}(V \ll c$ and $r \gg \alpha)$ denote the approximation of the Lagrange-Einstein function $L_{G}$ of a $P(m, q)$ submitted to the field created by this $P\left(m_{0}, q_{0}\right)$ where $\alpha$ denotes a certain length such that, in the static case it is simply $\alpha_{T}$. From the consideration of Sec. V of Zareski (2014), or of Eq. (9.23) of Zareski (2015), and of Eqs.

(9.1)-(9.11) of Zareski (2015) it follows that in this case, one has

$$
L_{G}(V \ll c \text { and } r \gg \alpha)=-m c \sqrt{c^{2}-V^{2}}+\frac{m c}{2} \alpha_{T} W_{\mu} \dot{x}^{\mu},
$$

where $\alpha_{T}$ is defined in Sec. 3.2. One sees that when $\boldsymbol{V}_{\mathbf{0}}=0$, then Eq. (57) becomes Eq. (25).

Conclusion regarding Maxwell's electrodynamics: Since, when $\boldsymbol{V}_{\mathbf{0}}=\mathbf{0}$ and when one imposes theoretically $k=0$ then Eq. (57) becomes Eq. (14) with $\alpha_{E}$ instead of $\alpha$, it follows that (57) produces only an approximation of the exact motion equation of the $P(m, q)$ submitted to the field created by the charge $q_{0}$ of velocity $\boldsymbol{V}_{\mathbf{0}}$. It appears therefore that Maxwell's electrodynamics theory is a non-relativistic approximation of the exact electrodynamics theory, and the Lienard-Wiechert potential $A_{\mu}$ defined by

$$
A_{\mu}=-\left[q_{0} /\left(4 \pi \varepsilon_{0}\right)\right] W_{\mu} .
$$

yields only an approximation of the motion equation.

Conclusion regarding classical gravitation: Since, when $\boldsymbol{V}_{\mathbf{0}}=\mathbf{0}$, and $q=0$, Eq. (57) becomes Eq. (14) which is an approximation of Eq. (3), it follows that (57) can produce only an approximation of the exact motion equation of the $P(m, q)$ submitted to the field created by the mass $m_{0}$ of velocity $\boldsymbol{V}_{\mathbf{0}}$. This confirms as we know that classical gravitation is an approximation of the relativistic gravitation, i.e., the gravitational Lienard-Wiechert potential $G_{\mu}$ defined by

$$
G_{\mu} \equiv m_{0} k W_{\mu},
$$

yields only an approximation of the motion equation. One recalls that $G_{\mu}$ is a gravitational Lienard-Wiechert potential tensor seen at an observation point $\boldsymbol{R}_{o b}$ due to the particle of mass $m_{0}$ that moves with the velocity $\boldsymbol{V}_{\mathbf{0}}$, and $\mathrm{r}$ is the distance between the position of $m_{0}$ at the time t' where the signal was emitted and reaches the point $\boldsymbol{R}_{o b}$ at the time $\mathrm{t}$ such that $\left(t-t^{\prime}\right) c=r$. It follows that the Lagrange-Einstein function $L_{G G}(V \ll$ $c$ and $r \gg \alpha$ ) is obtained from (57) by inserting $q q_{0}=0$.

One sees the similarity of the effects of the ether perturbations on the $P(m, q)$ of velocity $V \ll$ $c$ and $r \gg \alpha$, due to the electric charge $q_{0}$ of velocity $\boldsymbol{V}_{\mathbf{0}}$ or due to the massive particle $m_{0}$ of same $\boldsymbol{V}_{0}$, that is to say, the similarity of the electromagnetic Lienard-Wiechert potential tensor (59) with the gravitational tensor (60). But, Maxwell's equations do not take into account Einstein's General Relativity. That is to say that these equations are only an approximation for $V \ll c$ and $r \gg \alpha$ of the exact electromagnetism equations that takes into account general relativity.

4.2 Analogy of the Known Approximated Gravitation Tensor due to a $\mathbf{P}\left(\boldsymbol{m}_{\mathbf{0}}, \mathbf{0}\right)$ of Given Motion with the Electromagnetic Tensor due to an Electric Charge of Same Motion

Let $g_{\mu \nu}$ be the gravitational field created by $P\left(m_{0}, o\right)$ of given velocity $\boldsymbol{V}_{\mathbf{0}}$ to which is submitted a $P(m, 0)$ of velocity $\boldsymbol{V}$. What we know about these components $g_{\mu \nu}$ is that: from Eqs. (7) and (60), one deduces that, far from the source that creates the field $g_{\mu \nu}$, and for not large velocity of $P(m, q)$ in front of $c$, we know the approximate values of the $g_{\mu 4}$ denoted specifically by $g_{\mu 4}(V \ll c$ and $r \gg \alpha)$ for which the expression is, considering (60),

where $\delta_{v \mu}$ is the Kronecker delta.

$$
g_{4 \mu}(V \ll c \text { and } r \gg \alpha)=\delta_{4 \mu}-\left(\delta_{4 \mu}+1\right) m_{0} k W_{\mu} / c^{2}
$$


Indeed, Cf. Zareski (2014) \& Zareski (2015), when a $P(m, 0)$ is submitted to a gravitational field $g_{\mu \nu}$, we consider $L_{G}$ defined by (1) and (2), now $g_{\mu \nu} \dot{x}^{\mu} \dot{x}^{\nu}$ can be written

where $\Delta_{i \neq j}$ is defined by

$$
g_{\mu \nu} \dot{x}^{\mu} \dot{x}^{v}=g_{\mu \mu} \dot{x}^{\mu} \dot{x}^{\mu}+2 g_{4 j} \dot{x}^{4} \dot{x}^{j}+2 \Delta_{i \neq j},
$$

$$
\Delta_{i \neq j} \equiv g_{12} \dot{x}^{1} \dot{x}^{2}+g_{13} \dot{x}^{1} \dot{x}^{3}+g_{23} \dot{x}^{2} \dot{x}^{3}
$$

and $g_{\mu \mu}$, as following

$$
g_{\mu \mu}=g_{0, \mu \mu}+\delta g_{\mu \mu}
$$

where $g_{0, \mu \mu}$ denotes the free value of $g_{\mu \mu}$. With these notations (62) can be written:

and (1) as following

$$
g_{\mu \nu} \dot{x}^{\mu} \dot{x}^{v}=c^{2}-V^{2}+\delta g_{\mu \mu} \dot{x}^{\mu} \dot{x}^{\mu}+2 g_{4 j} \dot{x}^{4} \dot{x}^{j}+2 \Delta_{i \neq j}
$$

$$
L_{G}=-m c \sqrt{c^{2}-V^{2}}-m c \frac{\left(\delta g_{\mu \mu} \dot{x}^{\mu} \dot{x}^{\mu}+2 g_{4 j} \dot{x}^{4} \dot{x}^{j}+2 \Delta_{i \neq j}\right)}{2 \sqrt{c^{2}-V^{2}}}+\cdots
$$

We now define the NA, $L_{G, N A}$ of $L_{G}$, considering (66), one sees that

where $G_{\mu}$ is the tensor defined by

$$
L_{G, N A}=-m c \sqrt{c^{2}-V^{2}}+m G_{\mu} \dot{x}^{\mu} / c
$$

That is to say where (61)is verified.

$$
G_{4} \cong-c^{2} \delta g_{44} / 2, \quad G_{j} \cong-c^{2} g_{4 j}
$$

Now, one sees that $G_{\mu}$ and $A_{\mu}$ differ only by a constant multiplicative coefficient, furthermore considering (57) one see that that these two coefficients are added therefore a more general form of $g_{4 \mu}(V \ll c$ and $r \gg \alpha)$ that takes into account gravitation and electromagnetism, will be denoted $\beth_{4 \mu}(V \ll c$ and $r \gg \alpha)$, for which the expression is

$$
\mathrm{Z}_{4 \mu}(V \ll c \text { and } r \gg \alpha)=\delta_{4 \mu}-\left(\delta_{4 \mu}+1\right) \alpha_{T} W_{\mu} / 2 .
$$

Now since in absence of electromagnetism the $g_{4 \mu}(V \ll c$ and $r \gg \alpha)$ are the approximation of $g_{4 \mu}$, it follows that the $\beth_{4 \mu}(V \ll c$ and $r \gg \alpha)$ are the approximations of the exact components $\beth_{4 \mu}$ of the field $\beth_{v \mu}$ which is the exact solution of Einstein's general relativity equations, and since the $\beth_{4 \mu}(V \ll c$ and $r \gg \alpha)$ differ from the components $g_{\mu 4}(V \ll c$ and $r \gg \alpha)$ only by a constant coefficient, i.e., by the fact that $m_{0} k / c^{2}$ becomes $\alpha_{T} / 2$, it follows one can determine a tensor of components $\beth_{v \mu}$ such that these components will differ from the components $g_{\mu \nu}$ only by the fact that $m_{0} k / c^{2}$ will becomes $\alpha_{T} / 2$; therefore this tensor $\beth_{v \mu}$ is a solution of Einstein's general relativity equations.

\section{Conclusion}

It appears that Maxwell's electromagnetism can be generalized as to be a case of the General Relativity, that is, the exact Lagrange-Einstein function of an electric charge $q$ submitted to the field due an immobile charge $q_{0}$ and its motion equation are the same as those of a particle of mass $m$ submitted to only the field created by an immobile particle of mass $m_{0}$. In particular, it appears that an immobile $q_{0}$ creates also an electromagnetic horizon that behaves like a Schwarzschild horizon on which the velocity is null. Then that it exists ether gravitational waves constituted by gravitons in the same way as electromagnetic waves are constituted by photons.

Then we arrive to the conclusion that, since in the approximation $V \ll c, A_{\mu}$ and $G_{\mu}$ differs by only a constant multiplicative coefficient and since as we show, $G_{\mu}$ produces then the approximation of $g_{\mu 4}$, it follows that $A_{\mu}+$ $G_{\mu}$ produces the approximation for $V \ll c$ of $\beth_{\mu 4}$, where the $\beth_{\mu \nu}$ are the components of a tensor that generalizes Einstein's fundamental tensor $g_{\mu \nu}$ by taking into account the contribution of the electrical charge $q_{0}$ whether it moves or not.

\section{Acknowledgements}

I thank Professor Lawrence Horwitz from Tel Aviv University for his advice and encouragement.

\section{References}

Dirac, P. A. M. (1975). General Theory of Relativity. John Wiley \& Sons, Inc. 
Einstein, A. (1916). The Foundation of the General Theory of Relativity. Annalen der Physik, 49, 1916. reprinted in Lorentz, Einstein, Minkowski \& Weyl, here below precised, VII, pp. 109-164.

Einstein, A. (1920). Ether and the theory of relativity: The collected papers of Albert Einstein. Berlin: Springer.

Lorentz, H. A., Einstein, A., Minkowski, H., \& Weyl, H. (1923). The Principle of Relativity: A Collection or Original Memoirs on the Special and General Theory of Relativity. Dover.

Maxwell, J. C. (1954). A Treatise on Electricity and Magnetism. New-York: Dover.

Zareski, D. (2001). The elastic interpretation of electrodynamics. Foundations of Physics Letters, 14(5), 447-469.

Zareski, D. (2012). On the elasto-undulatory interpretation of fields and particles. Physics Essays, 25(2).

Zareski, D. (2013). Fields and wave-particle reciprocity as changes in an elastic medium: The ether. Physics Essays, 26(2), 288-295.

Zareski, D. (2014). The ether theory as implying that electromagnetism is the Newtonian approximation of general relativity. Physics Essays, 27(4), 517-522.

Zareski, D. (2015). Unification of Physics by the Ether Elasticity Theory. LAP LAMBERT Academic Publshing, 102 pages.

Zareski, D. (2016). On particle waves solutions of the equation of the ether. Journal of Advances in Physics (JAP), $9(2)$.

\section{Copyrights}

Copyright for this article is retained by the author(s), with first publication rights granted to the journal.

This is an open-access article distributed under the terms and conditions of the Creative Commons Attribution license (http://creativecommons.org/licenses/by/4.0/). 